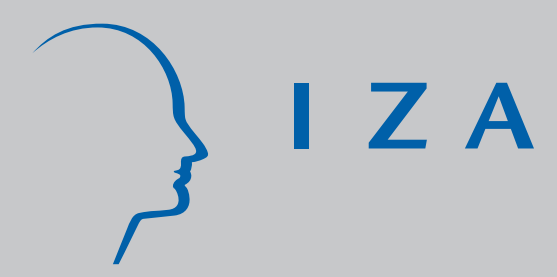

IZA DP No. 1286

Cut-Point Shift and Index Shift in Self-Reported Health

Maarten Lindeboom

Eddy van Doorslaer

September 2004 


\title{
Cut-Point Shift and Index Shift in Self-Reported Health
}

\author{
Maarten Lindeboom \\ Free University of Amsterdam, \\ Tinbergen Institute and IZA Bonn \\ Eddy van Doorslaer \\ Erasmus University Rotterdam
}

Discussion Paper No. 1286

September 2004

\author{
IZA \\ P.O. Box 7240 \\ 53072 Bonn \\ Germany \\ Phone: +49-228-3894-0 \\ Fax: +49-228-3894-180 \\ Email: iza@iza.org
}

Any opinions expressed here are those of the author(s) and not those of the institute. Research disseminated by IZA may include views on policy, but the institute itself takes no institutional policy positions.

The Institute for the Study of Labor (IZA) in Bonn is a local and virtual international research center and a place of communication between science, politics and business. IZA is an independent nonprofit company supported by Deutsche Post World Net. The center is associated with the University of Bonn and offers a stimulating research environment through its research networks, research support, and visitors and doctoral programs. IZA engages in (i) original and internationally competitive research in all fields of labor economics, (ii) development of policy concepts, and (iii) dissemination of research results and concepts to the interested public.

IZA Discussion Papers often represent preliminary work and are circulated to encourage discussion. Citation of such a paper should account for its provisional character. A revised version may be available directly from the author. 
IZA Discussion Paper No. 1286

September 2004

\section{ABSTRACT \\ Cut-Point Shift and Index Shift in Self-Reported Health*}

There is a concern that ordered responses on health questions may differ across populations or even across subgroups of a population. This reporting heterogeneity may invalidate group comparisons and measures of health inequality. This paper proposes a test for differential reporting in ordered response models which allows us to distinguish between cut-point shift and index shift. The method is illustrated using Canadian National Population Health Survey data. The McMaster Health Utility Index (HUI) is used as a more objective health measure than the simple 5-point scale of self-assessed health. We find clear evidence of index shifting and cut-point shifting for age and gender, but not for income, education or language.

JEL Classification: D30, D31, I10, I12

Keywords: hierarchical ordered probit, health measurement, cut-point shift, index shift, Canada

Corresponding author:

Maarten Lindeboom

Department of Economics

Free University of Amsterdam

De Boelelaan 1105

1081 HV Amsterdam

The Netherlands

Email: mlindeboom@econ.vu.nl

\footnotetext{
* This paper derives from the project "The dynamics of income, health and inequality over the life cycle" (known as the ECuity III Project), which is funded in part by the European Community's Fifth Framework Programme (contract QLK6-CT-2002-02297). We are grateful to the EC for financial support, to Statistics Canada for access to the NPHS data and to Pedro Pito Barros, Andrew Jones, Owen O'Donnell and participants of the ECuity III Workshop in Belgirate (Italy) and of the $4^{\text {th }}$ Health Economics Workshop in Oslo (Norway) for comments on an earlier version of the paper.
} 


\section{Introduction}

One of the most commonly employed indicators of overall individual health in general population surveys is the simple question "How is your health in general?", with response categories ranging from "very good" or "excellent" to "poor" or "very poor". While this ordinal variable is known to be a very good predictor of other outcomes, like subsequent use of medical care or mortality, there is some concern that its comparability across populations - or population subgroups is problematic because of a problem which has been termed 'state-dependent reporting bias' (Kerkhofs and Lindeboom, 1995), 'scale of reference bias' (Groot, 2000), 'response category cutpoint shift' (Sadana et al, 2000, Murray et al, 2001), 'reporting heterogeneity' (Shmueli, 2002, 2003) or 'differential item functioning' (Hays et al, 2000). ${ }^{1}$ Basically, it occurs if sub-groups of a population use systematically different threshold levels when assessing their health, despite having the same level of 'true' health. These differences may be influenced by, among other things, age, sex, education, language and personal experience of illness. It means that different groups appear to 'speak different languages' and to use different reference points when they are responding to the same question.

The problem may be particularly pronounced for comparisons across cultural groups with different norm and expectations. For example, Aboriginals in Australia report better self-assessed health (SAH) than the general Australian population while all other indicators (such as mortality) show this subgroup to be at a serious disadvantage with respect to health (Murray et al, 2001). Similarly, Sen (2002) recently quoted the well-known evidence from India again, where Kerala, the state with the highest life expectancy, consistently shows the highest rates of reported morbidity. He concludes that: "There is a strong need for scrutinising statistics on self reported illness in a social 
context by taking note of levels of education, availability of medical facilities and public information on illness and remedy". The best way to do this is to formalise the problem of heterogeneous reporting behaviour and to formulate tests for its occurrence in the context of subjective health information. This is the aim of this paper.

We present a framework for individual reporting behaviour that enables us to formally test whether variations in responses to health questions reflect true health differences or reporting behaviour. Our health-reporting model also allows us to distinguish between two types of reporting heterogeneity: cut-point shift and index shift. The latter refers to the situation where reporting behaviour leads to a in parallel shift of the thresholds that determine the response categories, so that their relative position remains unaltered. In the case of cut-point shift the thresholds are affected differently by the response behaviour, leading to a change of the relative positions of the reporting thresholds.

Heterogeneous reporting behaviour may have important implications for the measurement and explanation of inequalities in health by, for instance, income or education. If, given the same level of 'true' health, the assessment of reported health differs systematically by socio-economic status, this may bias the measured degree of socio-economic inequality in health. The distinction between cutpoint shift and index shift becomes relevant when one aims to unravel and quantify the contributions of various determinants of health to measured inequality. We will return to this in the final section and conclude that estimates of our reporting model can be used to obtain more reliable estimates of, for instance, inequality in health.

As will be clear intuitively, a valid benchmark that can be used to validate the responses to the SAH question is essential to our approach. The WHO's Evidence for Health Policy group ( Tandon et al, 2002) has proposed the use of vignettes, whereby respondents are asked to not only rate their own health state (or its dimensions) but to evaluate also health state descriptions of fictitious

\footnotetext{
1 There is also some concern about the reliability of responses to the self-assessed health question (Crossley and Kennedy, 2002), which relates to another type of measurement error than the one considered here.
} 
individuals. The health state evaluations of these fictitious individuals are used to estimate reporting thresholds (cut-points) that may depend on individual characteristics, and which are then used also to rate the individual's own health. ${ }^{2}$ An obvious alternative is to use a more objective indicator of health and to condition on this measure when making comparisons across different socio-economic groups. This is the route that we will follow in this paper. The vignette approach has its advantages, but it is very demanding in terms of data collection and very often not a realistic option for researchers wanting to analyze existing surveys. Moreover, as we will discuss in more detail at the end of section 2 , it is most likely that the implementation of the vignette approach also requires a more objective source of information about the respondents' health.

The approach adopted in this paper is inspired by Kerkhofs \& Lindeboom (1995), who focused on differential reporting behaviour across labour market states ${ }^{3}$. In this paper we focus on differential response to SAH by other background characteristics. We exploit the fact that the Canadian National Population Health Survey 1994-95 is one of the few general population health surveys which contains both the simple SAH question and one of the well-known generic measures of health utility. This is the McMaster Health Utility Index Mark III, which is used, inter alia, for computing healthy life expectancies in Canada (Feeny et al., 1995, Furlong et al., 1998). While this measure also relies on self-reporting, one advantage is that respondents are only required to classify themselves on eight health attributes. The overall individual health utility score on a scale of zero to one is derived using weights which are derived from a different valuation survey on a different sample of individuals. As such, it represents a more valid and reliable general health measure than the single SAH question.

2 A similar approach is taken by Van Doorslaer and Jones (2003) when they impose the thresholds obtained from the empirical cumulative distribution function of HUI to run interval regressions on self-assessed health.

3 There is an issue in the retirement literature that subjective health measures may depend on the retirement status of the respondent. Individuals out of work may overstate health problems in order to justify inactivity or because receipt of social security benefits requires ill health. Kerkhofs and Lindeboom (1995) formulate a health-reporting model that allows for differential reporting across labour market status (state dependent reporting behaviour). They found that Dutch recipients of Disability Insurance benefits strongly overstated their health problems. 
Ideally, one would prefer to use generic measures like the HUI to measure health differences across different socio-economic groups. However, such measures are rarely available in general social household surveys and when they are, they often differ across surveys, which makes comparisons across different populations impossible. By contrast, the general SAH question is very widely used. Performing tests for reporting heterogeneity in SAH using surveys with more abundant health information (such as the NPHS) provides information on the likelihood and size of the reporting heterogeneity problems in other surveys with simpler health measures ${ }^{4}$.

The paper is organised as follows. Section 2 presents the theoretical model of reporting behaviour which underlies our testing strategy. We use a Canadian data set (described in section 3) to illustrate the reporting model and the testing procedures and report on this in section 4 . We conclude in section 5. This section also contains a discussion on how the reporting model could be used for the measurement and decomposition of health inequality.

\section{A model of reporting behaviour}

The reported subjective health measure is denoted by $H^{S}$ and refers to the respondent's answer to a question like "How good is your health in general?", with replies ranging from excellent, very good, good, fair, to poor. It is assumed that these responses are generated by a corresponding latent true health variable $H^{*}$. Rather than one single index, $H^{*}$ could refer to a set of latent health indices covering the different domains of individual health. For ease of exposition we will refer to it as a single health index. The reporting model relates the unobserved latent health to the individual's responses to the health question. Important for the issue of cross-population comparability is that the relationship between $H^{*}$ and $H^{S}$ may not be constant across populations and may even vary within populations. For instance, an

\footnotetext{
4 Simpler measures are, for instance, ADL measures, or whether or not the respondent suffers from a range of chronic conditions. On its own, each of these measures may not sufficiently well describe the general health status of an individual and it will be difficult to use the different measures simultaneously to describe health
} 
individual with a university degree and a true health level $\hat{H}^{*}$ may state that his health is 'fair', whereas an otherwise identical but lower educated respondent may report to be in excellent health. Or stated more generally: the response labels may have a different meaning in different subgroups of the population.

The concept of true health $H^{*}$ is essential for the issue of cross-population comparability. Any unconditional comparison of $H^{S}$ (i.e. not controlling for $H^{*}$ ) across different groups of the population may reflect both differences in health conditions and differences in reporting behaviour. In practical situations one does not observe $H^{*}$, but only a range of more objective measures. For example, whether an individual suffers from various health problems, diseases and health-related impediments in performing a range of daily activities. These could be used to proxy the (different domains of) true unobserved health $\left(H^{*}\right)$.

The above results in the following equations of the health reporting model:

$$
\begin{aligned}
& H^{S}=f_{1}\left(H^{*}, X_{1}, \varepsilon_{1} ; \beta_{1}\right) \\
& H^{*}=f_{2}\left(H^{o}, X_{2}, \varepsilon_{2} ; \beta_{2}\right)
\end{aligned}
$$

The variables $\varepsilon_{1}$ and $\varepsilon_{2}$ are random variables, $f_{1}($.$) describes the reporting behaviour and f_{2}($.$) the$ relationship between true health and its determinants. Consequently, the effect of $X_{1}$ (as embodied in the $\beta_{1}$ 's) can be viewed as pure reporting behaviour, whereas $X_{2}$ corrects for the dissimilarity between $H^{o}$ and $H^{*}$. Note that $f_{2}$ will be an identity in the (ideal) case where $H^{*}$ is fully captured by $H^{o} . H^{*}$ is by definition unobserved. The difficulty lies in the assessment of the relative importance of $X_{1}$ (reporting behaviour) versus $X_{2}$ (true health effects or heterogeneity in health) in the determination of $H^{S}$. It will depend crucially on how the empirical model is implemented.

We will often observe $H^{S}$ as an ordered categorical variable. The essence of differential reporting is that $X_{1}$ will possibly not only affect the mean of the index function, but will, in addition, 
have a direct effect on the cut-points corresponding to the different response categories. $X_{2}$ is included to measure the dissimilarities between $H^{o}$ and $H^{*}$ and therefore its role is expected to be different. More specifically, the health response is defined as:

$$
H^{S}=i \Leftrightarrow \quad c_{i-1}<H^{*} \leq c_{i,}, i=1, . ., n
$$

Where $n$ is the number of response categories. The cut-points $c_{i}$, are allowed to vary with different values of $X_{1}$ :

$$
c_{i,}=g_{i}\left(X_{1} ; \beta_{1 i}\right), \quad i=1, \ldots, n-1, c_{0}=-\infty, c_{n}=\infty .
$$

In this model there may be differential responses on $H^{S}$ for respondents with identical levels of true health $\left(H^{*}\right)$ and this is captured by $g_{i}\left(X_{1} ; \beta_{1 i}\right)$. The function $g_{i}($.$) is left unspecified, but in its most$ flexible form each cut-point is affected by exogenous variables in a different way ( $\left.\beta_{1 i}, i=1, \ldots, n-1\right)$. The latent level of true health $H^{*}$ is unobserved and if we assume that we can approximate it with :

$$
H^{*}=f\left(H^{o} ; \alpha\right)+X_{2} \beta_{2}+\varepsilon_{2}
$$

then we obtain the empirical counterpart of the reporting equation (2a):

$$
H^{S}=i \Leftrightarrow \quad g_{i-1}\left(X_{1} ; \beta_{1 i-1}\right)-X_{2}^{\prime} \beta_{2}<f\left(H^{o} ; \alpha\right)+\varepsilon_{2} \leq g_{i}\left(X_{1} ; \beta_{1 i}\right)-X_{2}^{\prime} \beta_{2}
$$

Equation (3a) is informative on the role of $X_{1}$ and $X_{2}$ and can serve as a basis for tests for different types of reporting behaviour. Reporting heterogeneity may affect the mean and the shape of the SAH distribution. A particular type of reporting heterogeneity is that $X_{1}$ shifts all thresholds $c_{i}$ in parallel. This is equivalent to a shift of the index function that generates the discrete responses $H^{S}$ and we therefore refer to this kind of reporting behaviour as index shift reporting. Alternatively, in the case that $X_{1}$ affects the thresholds in a dissimilar way, we speak of cut-point shift reporting. As an example, all thresholds could shift in parallel if in a certain cultural group all respondents are typically more modest about their health evaluation. Or if due to peer group effects the general perception of health changes. Cut-point shift is relevant, if for instance, in a specific culture it could be considered to be improper to use the word "excellent" to denote one's situation in general, or one's health status in particular, and that 
would exclusively raise the threshold for the excellent health category. Disability benefits are contingent upon bad health, which may provoke these respondents to report poor health, regardless of their true level of health ('State-dependent' reporting behaviour, Kerkhofs \& Lindeboom 1995).

The distinction between the two types of reporting behaviour can be tested directly by imposing the restriction that the $\beta_{l i}$ are equal for all $i$. Rejection of the null hypothesis implies differential effects of $X_{1}$ on the reporting thresholds and hence cut-point shift in reporting. However, under the null hypothesis, the matter is less straightforward. In this case only the difference between $X_{1}$ and $X_{2}$ is identified and we therefore cannot distinguish whether an effect of an exogenous variable reflects index shift reporting $\left(X_{1}\right)$ or true health effects $\left(X_{2}\right)^{5}$. This is a fundamental identification problem that cannot be resolved without external information or arbitrary exclusion restrictions ${ }^{6}$. It will depend on the empirical application whether either of these two identifying assumptions can be applied. Indeed the empirical implementation of the model and the measure(s) used to approximate $H^{*}$ will be important.

Our empirical strategy will be to estimate separate ordered response models for groups in our sample stratified according to age, sex, education, income and language. More specifically:

$$
H_{k}^{S}=i \Leftrightarrow \quad \delta_{i-1}^{k}<f\left(H^{o} ; \alpha^{k}\right)+\varepsilon_{2}^{k} \leq \delta_{i}^{k}
$$

Note that this specification is more flexible than the usual hierarchical ordered response models like e.g. the HOPIT model. In these models one usually assumes a common vector $\alpha$ and imposes a functional form on the function $g_{i}$. For instance, Kerkhofs \& Lindeboom (1995), Groot (2000) and Tandon et al (2002) take $g_{i}\left(X_{1} ; \beta_{1 i}\right)=X_{1}{ }^{\prime} \beta_{1 i}$. In our specification of (3b), each subgroup $k$ is allowed to differ both with respect to cut-points ( $\delta_{i}$ 's) and with respect to index effects $(\alpha \text { 's })^{7}$.

\footnotetext{
5 Over and above the effect of $H^{\circ}$.

6 This holds for all models that rely on measures for $H^{o}$ to identify reporting heterogeneity, such as Shmueli (2003), but to a lesser extent for models that use a vignette approach (Tandon et al 2002). We turn to these models below.

7 The separate $\delta$ 's in our specification do not impose the linear additive functional form and allow for interaction effects of all elements of $X_{1}$.
} 
A test for differential response behaviour across subgroups, i.e. different $\delta_{i}$ 's and/or different $\alpha$ 's, can be based on straightforward likelihood ratio tests. The tests compare the sum of the likelihood values for two subgroups $k$ and $k$ ' with the likelihood values obtained using the total group (i.e. with equal $\delta_{i}$ 's and $\alpha$ 's imposed). In cases where equality of response behaviour for two groups is rejected, we can additionally test whether this is due to index shifts (different $\alpha$ 's) or to cut-point shifts (different $\delta_{i}$ 's). This can be done by estimating (3b) subject to either the restriction that $\delta_{i}^{k}=\delta^{k^{\prime}}{ }_{i}$ or $\alpha^{k}=\alpha^{k^{\prime}}, \forall i$, and to compare the likelihood value of the restricted model $\left(\Lambda^{\mathrm{R}}\right)$ with the sum of the likelihood values of the unrestricted models $\left(\Lambda^{\mathrm{U}}\right)$. A test is based on $-2 *\left(\Lambda^{\mathrm{R}}-\Lambda^{\mathrm{U}}\right)$ which is $\chi^{2}$ distributed with degrees of freedom equal to the number of restricted parameters. Below we will illustrate these testing procedures with data from the Canadian National Population Health Survey 1994-95. Before we do this we would like to make three remarks.

First, the core of the model is equation (1) and the equations that relate this model to the actual responses on the SAH question (equations 2 and 1b'). This seems the most natural way to implement the reporting behaviour. Given the model structure, the testing procedure remains identical, regardless of the estimation method used and as such it is immaterial whether one uses hierarchical response, interval regression or other methods to estimate the parameters in (3a) or (3b).

Secondly, the response equation (3a) with individual-specific thresholds can also be derived from an ordered response model with heteroskedastic error terms, regardless of whether there exist variations in cut-points across different socio-economic groups (see van Doorslaer and Jones (2002)). This only affects the interpretation of the cut-point functions $g_{i}\left(X_{1} ; \beta_{1 i}\right)$ and it does not influence our testing procedure to distinguish between cut-point shift and index shift.

Thirdly, essential to our approach is a valid benchmark: the objective health measure $H^{o}$. Tandon et al (2002) have proposed the use of vignettes to make the self-report responses comparable across different populations. The vignettes are included in the WHO Multi-Country Study and are designed to calibrate the six domains of the WHO generic health measure. For each domain, like e.g. mobility, respondents are asked to rate the degree of impairment for descriptions of fictitious 
individuals. The descriptions of these individuals range from a perfectly healthy athlete to an almost fully paralysed individual. Respondents are asked to classify these vignettes on a similar five-point response scale as the SAH measure. Differences in the response behaviour across different subpopulations are then used to identify variations in the cut-points. More specifically, Tandon et al (2002) model the responses to vignettes $k, Y^{k}$, as:

$$
Y^{k}=i \quad \Leftrightarrow \quad c_{i-1}<Y^{*} \leq c_{i,}, i=1, . ., n
$$

With the response thresholds as $c_{i}=g_{i}\left(X_{1} ; \beta_{1 i}\right)=X_{1}{ }^{\prime} \beta_{1 i}$ and the index function as $Y^{*}=X_{2}{ }^{\prime} \beta_{2}+$ $\varepsilon, \varepsilon \sim N(0,1)$.

A first observation regarding this model is that, as for our model, it is not clear which variables belong in the thresholds $\left(X_{1}\right)$ and which variables in the index function $\left(X_{2}\right)$. For this choice one has to rely on a testing procedure like the one we proposed above to distinguish between cut-point shift and index shifts. Secondly, the rating of a specific health situation may also depend on the respondents's own health status. It is not unlikely that the view of very fit and healthy individuals on specific health state descriptions will differ from the views of individuals with severe physical impairments. If this is the case, then one should add the respondent's health status as a conditioning variable in $X_{2}$. We then have, in essence, returned to our model summarised in (3a) and all caveats listed above hold, except the remark on the distinction between index shift and true health differences and the interpretation of $\beta_{2}$. In the vignette model all variation can be ascribed to differential reporting behaviour ${ }^{8}$.

\section{Data and variable definitions}

The data used in this paper are taken from the first wave (held in 1994-1995) of the Canadian National Population Health Survey (NPHS) (Tambay and Catlin, 1995). For our analyses we have included respondents aged between 20 and 70 years and have excluded cases with incomplete or

\footnotetext{
8 The vignettes can be viewed as an external source of information concerning the role of the exogenous variables.
} 
inconsistent information on the relevant socio-demographic and health variables. The remaining sample size consists of 13,699 observations.

The two key variables for this study are self-assessed health (SAH) and the more objective health status as measured by the Health Utility Index (HUI). In terms of the notation of the previous section, $H^{S}$ equals SAH and $H^{O}$ equals HUI. In the $N P H S$, respondents were asked: "In general, how would you say your health is?" The response categories were excellent, very good, good, fair, and poor. Because only a relatively small fraction of the sample is in poor health, we combine the poor and fair category of the SAH variable. Also, each respondent was assigned a Health Utility Index score based on the responses to the questions of the eight-attribute Health Utility Index Mark III health status classification system. The Health Utility Index is a generic health status index, developed at McMaster University that measures both quantitative and qualitative aspects of health (Torrance et al, 1995, 1996; Feeny et al, 1995). It provides a description of an individual's overall functional health, based on eight attributes: vision, hearing, speech, ambulation, dexterity, emotion, cognition, and pain. The Health Utility Index assigns a single numerical value, between zero and one, for all possible combinations of levels of these eight self-reported health attributes. A score of one indicates perfect health. The Health Utility Index also embodies the views of society concerning health status, inasmuch as preferences about various health states are elicited from a representative sample of individuals. In this way, indirectly the HUI also embodies information based on the valuation of vignettes, such as the ones proposed by Tandon et al (2002), but respondents are asked to value all health domains simultaneously into a single utility index. This makes the HUI a very suitable candidate for the more objective health variable where we need to condition on $\left(H^{O}\right)$. More specifically, with HUI included, the role of $X_{2}$ in the model is expected to be modest.

Total income before taxes and deductions is measured in the NPHS as a categorical variable with 11 response categories. Education is measured as the highest level of general or higher education completed (5 levels). Table A1 provides some descriptive statistics of the relevant (but dichotomized) variables in our analysis. Reporting behaviour may differ across socio-economic status, but also across different cultural or ethnic groups. The Canadian NPHS can to some extent be used to test for 
cross-cultural differences in reporting behaviour by using language as a proxy for groups with different cultural backgrounds. We distinguish four language groups: those speaking English only, French only, those speaking both languages and those speaking another language only.

Table A2 provides cross-tabulations of mean HUI values by level of SAH and background characteristics. The tabulations show that, on average, the male, the younger, and the higher education and income respondents report higher health utility values, but conditional on reported level of SAH, the differences are much smaller. Only at lower levels of health, some differences remain, with particularly the female, older, and lower income respondents reporting lower mean HUI values than their counterparts. In the next section, we explain how we have formally tested for reporting heterogeneity.

\section{Illustration of the method: empirical implementation and results}

\section{Empirical implementation}

We apply the procedure discussed in section 2 to the Canadian NPHS data. Estimation of model (4') requires us to be more specific about the functional form of $f\left(H^{o} ; \alpha^{k}\right)$ and the distribution of $\varepsilon_{2}^{k}$. We take $f\left(H^{o} ; \alpha^{k}\right)$ as a quadratic function of the generic overall health status index (the Health Utility index, HUI). $\varepsilon_{2}^{k}$ is assumed to have a standard normal distribution. Note that our tests for differential response and the distinction between cut-point and index shift do not depend on these assumptions.

The strategy proposed in section 2 is to stratify the sample into (more) homogenous subgroups and to estimate ordered response models for each group. We stratify the sample according to language, age, gender, income and education. For each group, we obtain estimates of $\alpha^{k}$ (index function) and $\delta_{I}^{k}$ (cut-points). We then aggregate over one of the stratifiers and estimate a model with the stratification variable included in the index function. This model assumes that there is a common effect of $f\left(H^{o} ; \alpha^{k}\right)$ and that there are common cut-points. 
For example, we observe four different language (or cultural) groups in the sample: French only (Fr), English only (En), French and English (Bo) and Other (Ot). We observe an indicator for objective health $H^{o}$ and a response to a question concerning general health $\left(H^{S}\right)$. The latter variable is an ordered categorical variable, with 4 response categories ${ }^{9}$. It is hypothesized that the different cultural backgrounds may lead to differential response behaviour, even if individuals have identical health $\left(H^{o}\right)$. So, according to the differential response model $(3 b)$ :

$$
H_{k}^{S}=i \Leftrightarrow \quad \delta_{i-1}^{k}<f\left(H^{o} ; \alpha^{k}\right)+\varepsilon_{2}^{k} \leq \delta_{i}^{k}
$$

For each $\mathrm{k}(\mathrm{Fr}, \mathrm{En}, \mathrm{Bo}, \mathrm{Ot})$ we obtain a separate set of estimates of $8\left(4^{*} 2\right) \alpha^{k}$ and $12(4 * 3) \delta^{k}{ }_{i}$ parameters. The null hypothesis of reporting homogeneity is that there is no differential response with respect to the index (for all $\mathrm{k}, \alpha^{k}=\alpha$; i.e. the translation of objective health into subjective health is identical across groups) and the cut-off points (for all $\mathrm{i}$ and $\mathrm{k}: \delta_{i}^{k}=\delta$; i.e. the response categories have the same meaning for all $k=\mathrm{Fr}$, En, Bo, Ot groups):

$$
H_{k}^{S}=i \Leftrightarrow \quad \delta_{i-1}<f\left(H^{o} ; \alpha^{\prime}\right)+\beta_{1} \mathrm{Fr}+\beta_{2} \mathrm{En}+\beta_{3} \mathrm{Bo}+\beta_{4} \mathrm{Ot}+\varepsilon_{2} \leq \delta_{i}
$$

In this specification, the language variables are still included in the index, but this is not required ${ }^{10}$. Our test for differential response behaviour is based on the comparison of the likelihood value of the restricted model with 8 parameters (3b'), with the unrestricted model with 20 parameters (3b'). If model (3b') is rejected (i.e., if homogeneous response behaviour proves invalid) a model can be estimated with the index function as in (3b"), but with differential (i.e. language-specific) cut-off points are allowed. A likelihood ratio comparison of this model with (3b") then can reveal whether the differential response is due to differential cut-points or to an index shift.

This procedure was applied for five stratifying variables: four language groups ( French, English, French and English and Other), two gender groups (male, female), two age groups (below

\footnotetext{
9 The original variable contains 5 response categories ranging from excellent to poor. In the empirical analyses we have merged the response categories "fair" and "poor".

10 Of course also a normalisation is required.
} 
and over 45), two educational groups (high and low), and two income groups (high and low). Low income is defined as an income less then Can $\$ 24.748$. A low education is defined as the highest level of educational attainment being secondary school or less.

\section{Results}

The likelihood test results based on subgroup estimations of the various models are presented in Tables 1-5. We start by testing for homogeneity in response behaviour by language. Language is the closest we could get with this sample to differences in cultural backgrounds in Canada, and it has been argued that response behaviour is likely to differ most with respect to culture. We distinguish four language groups: people who speak English only, French only, both of these or another language. We do test this separately for 16 groups distinguished by 4 dichotomizations of income, education, age and gender. It can be seen from Table 1 that homogeneity is rejected in only 4 cases, three groups of males and one of females. Where homogeneity was rejected, we also tested whether the rejection was due to index shift or cut-point shift. In all of the male groups, it was only due to index shift. Only in the group of old females with low education and income, there was clear evidence of cut-point shift.

$<$ include tables 1-5 around here>

Next we aggregated across language groups and tested for response shift by income, while controlling for language in the index function only. The test results in Table 2 show that in one group only (young males with low education), homogeneity across income was rejected, and it was due entirely to index shift. Table 3 presents the same results for education. It is striking to see that for none of the four demographic groups we find any evidence of response shift by education. Apparently, the relationship between HUI and SAH is similar for those with high and low education. All in all, for the two indicators of socioeconomic status, we find little or no evidence of response shift. 
The picture is quite different for age and gender, as can be seen from Tables 4 and 5 . We find a clear rejection of the reporting homogeneity hypothesis for both of these demographic characteristics. Given similar HUI levels, males and females, and young and old, do not report similar levels of SAH. In particular, younger and female respondents seem to rate their health levels lower than older and male respondents with a similar HUI. This is in line with the findings of Van Doorslaer \& Gerdtham (2003) for Swedish adults: they found that the predictive effect of SAH on subsequent survival did differ by age and sex, but not by income and education.

\section{Conclusion and discussion}

Differential health reporting by subgroups of the population presents a potentially serious problem to the validity of subgroup comparisons of health levels and degrees of inequality. Any tests for such differential reporting inevitably have to condition on some other, preferably more objective, measure of health. For many purposes, the collection of additional data using vignette-type questions will prove very data demanding and not feasible for general-purpose surveys. In such cases, one will have to rely on the use of other, more objective, health measures collected in the survey.

The paper shows how differential reporting by subgroups can be tested in the context of an ordered response model and how cut-point shift and index shift can be separated using simple likelihood ratio tests. However, in the case of index shift, it is not possible to distinguish reporting behaviour from true health effects. This is a fundamental identification problem that holds for all models that aim to identify reporting heterogeneity from true health effects and this problem cannot be solved without external information and/or arbitrary exclusion restrictions. An exception is the vignette approach suggested by Tandon et al (2002). We discuss how our approach relates to their approach and conclude that our testing procedure to distinguish between cut-point shift and index shift is also needed for the vignette method. We also argue that if the responses to the vignettes depend on the respondent's health status, as in our method, conditioning on other objective health measures may be required. 
We illustrate our model and testing procedure by examining whether in Canada the relationship between the ordered responses to the self-assessed health (SAH) question is prone to index and cut-point shift. For language, income and education we find very few violations of the homogeneous reporting hypothesis, and in the few cases where it is violated, it appears almost invariably due to index rather than cut-point shift. The results are very different for age and gender: males and females respond differently to the SAH question, and so do the young and the older respondents. Female and older respondents appear to be milder in their self-assessments than their male and younger counterparts. Moreover, the differences are reflected both in index and in cut-point shifts. As a result, any differences between these groups in SAH tend to understate the 'true' differences insofar as these are mirrored by the differences in the Health Utility Index.

It is possible, of course, that these heterogeneous responses by age and gender are due to the fact that the multi- and single attribute utility functions used to generate the individual HUI values were not estimated age and gender specific. Utility values associated with, for instance, a certain degree of mobility or vision, may indeed differ by age and gender. If such age/gender specificities in the utility functions are deemed legitimate, this could explain our findings. If, as in the WHO approach, both the item classification and the rating of vignettes are obtained from the same respondents in the same survey, then allowing age and gender to affect the vignette ratings will probably reduce the likelihood of rejecting the homogeneity hypothesis. But then the crucial question becomes what is to be defined as 'true health'. If, for instance, the elderly are more inclined to rate their general health as good despite a lower objective health level in terms of abilities than younger respondents, is this to be considered as "misreporting" or can it alternatively be considered acceptable that expectations are lowered with rising age and therefore 'true' health levels can be considered equal? This brings us to the boundaries between positive and normative economics. Are respondents' self-assessments to be respected or to be adjusted for some "average" population norm? The approach taken in this paper takes the latter stance, and indeed it is questionable whether any test for reporting heterogeneity can be conceived when the former stance is adopted. 
The results we have obtained are, of course, sample specific and do not necessarily hold for other populations than the adult Canadian population. Future research for other countries should make clear whether these results can be generalised. The tools provided in this paper are also relevant for the measurement and decomposition of inequalities in health (as in Van Doorslaer and Jones, 2003). In the case of index shifts, estimates of health inequalities or health differences between socioeconomic groups will not suffer from reporting heterogeneity as long as one can control for these differences, for instance via standardisation on the relevant covariates. In the case of cut-point shift such standardisation is less straightforward because it relates to the SAH measure in a non-linear fashion and other ways to control for reporting heterogeneity are called for. One option is to exploit the structure of the reporting model. Model estimates can be used to generate an estimate of the 'true' health $\left(\hat{H}^{*}\right)$ via equation (1b'). In this measure variations in cut-points are eliminated, as by construction of the model these are in the response category thresholds $\left(c_{i}\right)$. Next, one could use the index $\hat{H}^{*}$ to measure the degree of income related health inequalities. An illustration of this would go beyond the scope of the current paper and we therefore leave this for future research. 
Tables

Table 1 Test for differential response by language group

\begin{tabular}{|c|c|c|c|c|c|c|c|c|}
\hline \multirow[t]{3}{*}{ Males } & \multicolumn{4}{|c|}{ Young } & \multicolumn{4}{|c|}{ Old } \\
\hline & \multicolumn{2}{|c|}{ Education high } & \multicolumn{2}{|c|}{ Education low } & \multicolumn{2}{|c|}{ Education high } & \multicolumn{2}{|c|}{ Education low } \\
\hline & Income high & Income low & Income high & Income low & Income high & Income low & Income high & Income low \\
\hline log lik French speaking & -33.71 & -31.89 & -61.68 & -123.41 & -73.49 & -81.88 & -40.00 & -108.48 \\
\hline log lik English speaking & -600.24 & -277.09 & -449.53 & -490.61 & -942.05 & -602.32 & -454.03 & -559.29 \\
\hline $\log$ lik Both lanuages & -142.05 & -60.33 & -84.20 & -104.04 & -286.11 & -145.22 & -93.15 & -85.41 \\
\hline log lik Other language & -175.68 & -115.25 & -97.86 & -138.11 & -235.92 & -171.42 & -78.20 & -110.21 \\
\hline Log lik sum (unrestricted model, $\Lambda^{\mathrm{U}}$ ) & -951.69 & -484.56 & -693.28 & -856.17 & -1537.58 & -1000.84 & -665.38 & -863.38 \\
\hline Log likelihood restricted model $\left(\Lambda^{\mathrm{R}}\right)$ & -957.34 & -495.72 & -696.79 & -861.65 & -1548.93 & -1012.02 & -672.32 & -870.89 \\
\hline$\chi^{2}$ test for overall shift $-2 *\left(\Lambda^{\mathrm{R}}-\Lambda^{\mathrm{U}}\right)$ & 11.30 & 22.31 & 7.02 & 10.96 & 22.71 & 22.37 & 13.88 & 15.02 \\
\hline P-value (12 degrees of freedom) & 0.50 & 0.03 & 0.86 & 0.53 & 0.03 & 0.03 & 0.31 & 0.24 \\
\hline Log lik semi-restr model (cut-pnts free, $\Lambda^{\mathrm{CP}}$ ) & n.a. & -490.80 & n.a. & n.a. & -1546.89 & -1008.52 & n.a. & n.a. \\
\hline$\chi^{2}$ test for cut-point shift $-2 *\left(\Lambda^{\mathrm{R}}-\Lambda^{\mathrm{CP}}\right)$ & n.a. & 9.82 & n.a. & n.a. & 4.08 & 7.01 & n.a. & n.a. \\
\hline P-value ( 9 d.f.) & n.a. & 0.37 & n.a. & n.a. & 0.91 & 0.636 & n.a. & n.a. \\
\hline$\chi^{2}$ test for index shift $-2 *\left(\Lambda^{\mathrm{CP}}-\Lambda^{\mathrm{U}}\right)$ & n.a. & 12.49 & n.a. & n.a. & 18.63 & 15.36 & n.a. & n.a. \\
\hline P-value (6 d.f.) & n.a. & 0.05 & n.a. & n.a. & 0.00 & 0.02 & n.a. & n.a. \\
\hline \multirow[t]{3}{*}{ Females } & \multicolumn{4}{|c|}{ Young } & \multicolumn{4}{|c|}{ Old } \\
\hline & \multicolumn{2}{|c|}{ Education high } & \multicolumn{2}{|c|}{ Education low } & \multicolumn{2}{|c|}{ Education high } & \multicolumn{2}{|c|}{ Education low } \\
\hline & Income high & Income low & Income high & Income low & Income high & Income low & Income high & Income low \\
\hline log lik French speaking & -63.65 & -40.52 & -59.65 & -206.82 & -112.21 & -114.42 & -53.00 & -179.85 \\
\hline log lik English speaking & -704.39 & -386.92 & -446.10 & -755.92 & -1016.34 & -961.91 & -351.37 & -748.52 \\
\hline log lik Both languages & -150.87 & -64.94 & -69.15 & -162.07 & -362.63 & -252.63 & -66.11 & -135.06 \\
\hline log lik Other language & -132.94 & -110.11 & -113.91 & -201.85 & -214.36 & -229.30 & -40.83 & -140.71 \\
\hline Log lik sum (unrestricted model, $\Lambda^{\mathrm{U}}$ ) & -1051.85 & -602.49 & -688.80 & -1326.65 & -1705.55 & -1558.26 & -511.31 & -1204.14 \\
\hline Log likelihood restricted model $\left(\Lambda^{\mathrm{R}}\right)$ & -1056.05 & -607.99 & -695.15 & -1334.47 & -1709.37 & -1566.56 & -519.20 & -1217.68 \\
\hline $\mathrm{X}^{2}$ test for overall shift $-2 *\left(\Lambda^{\mathrm{R}}-\Lambda^{\mathrm{U}}\right)$ & 8.40 & 10.99 & 12.70 & 15.64 & 7.66 & 16.62 & 15.78 & 27.07 \\
\hline P-value & 0.75 & 0.53 & 0.39 & 0.21 & 0.81 & 0.16 & 0.20 & 0.01 \\
\hline Log lik semi-restr model (Cut-pnts free, $\Lambda^{\mathrm{CP}}$ ) & n.a. & n.a. & n.a. & n.a. & n.a. & n.a. & n.a. & -1206.17 \\
\hline $\mathrm{X}^{2}$ test for cut-point shift $-2 *\left(\Lambda^{\mathrm{R}}-\Lambda^{\mathrm{CP}}\right)$ & n.a. & n.a. & n.a. & n.a. & n.a. & n.a. & n.a. & 23.01 \\
\hline P-value (9 d.f.) & n.a. & n.a. & n.a. & n.a. & n.a. & n.a. & n.a. & 0.006 \\
\hline $\mathrm{X}^{2}$ test for index shift $-2 *\left(\Lambda^{\mathrm{CP}}-\Lambda^{\mathrm{U}}\right)$ & n.a. & n.a. & n.a. & n.a. & n.a. & n.a. & n.a. & 4.06 \\
\hline P-value (6 d.f.) & n.a. & n.a. & n.a. & n.a. & n.a. & n.a. & n.a. & 0.67 \\
\hline
\end{tabular}


Table 2 Test for differential response by income

\begin{tabular}{|c|c|c|c|c|c|c|c|c|}
\hline \multirow{3}{*}{$\begin{array}{l}\text { Grouped over language } \\
\text { (but controlled for in index) }\end{array}$} & \multicolumn{4}{|c|}{ Males } & \multicolumn{4}{|c|}{ Females } \\
\hline & \multicolumn{2}{|c|}{ Young } & \multicolumn{2}{|c|}{ Old } & \multicolumn{2}{|c|}{ Young } & \multicolumn{2}{|c|}{ Old } \\
\hline & Educ high & Educ low & Educ high & Educ low & Educ high & Educ low & Educ high & Educ low \\
\hline Log lik High income earners & $-957,34$ & $-696,79$ & $-1548,93$ & $-672,32$ & $-1056,05$ & $-695,15$ & $-1709,37$ & $-519,20$ \\
\hline Log lik Low income earners & $-495,72$ & $-861,65$ & $-1012,02$ & $-870,89$ & $-607,99$ & $-1334,47$ & $-1566,56$ & $-1217,68$ \\
\hline Log lik sum (unrestricted model, $\Lambda^{\mathrm{U}}$ ) & $-1453,05$ & $-1558,44$ & $-2560,95$ & $-1543,21$ & $-1664,04$ & $-2029,62$ & $-3275,94$ & $-1736,88$ \\
\hline Log likelihood restricted model $\left(\Lambda^{\mathrm{R}}\right)$ & $-1459,44$ & $-1563,34$ & $-2570,69$ & $-1544,78$ & $-1668,38$ & $-2035,27$ & $-3278,79$ & $-1738,98$ \\
\hline$-2 *\left(\Lambda^{\mathrm{R}}-\Lambda^{\mathrm{U}}\right)$ & 12,78 & 9,80 & 19,47 & 3,13 & 8,68 & 11,29 & 5,70 & 4,21 \\
\hline P-value ( 7 degrees of freedom) & 0,08 & 0,20 & 0,01 & 0,87 & 0,28 & 0,13 & 0,58 & 0,76 \\
\hline Log lik semi-restricted model (Cut-pnts free, $\Lambda^{\mathrm{CP}}$ ) & n.a. & n.a. & $-2570,60$ & n.a. & n.a. & n.a. & n.a. & n.a. \\
\hline $\mathrm{X}^{2}$ test for cut-point shift $-2 *\left(\Lambda^{\mathrm{R}}-\Lambda^{\mathrm{CP}}\right)$ & n.a. & n.a. & 0,18 & n.a. & n.a. & n.a. & n.a. & n.a. \\
\hline P-value ( 3 d.f.) & n.a. & n.a. & 0,98 & n.a. & n.a. & n.a. & n.a. & n.a. \\
\hline $\mathrm{X}^{2}$ test for index shift $-2 *\left(\Lambda^{\mathrm{CP}}-\Lambda^{\mathrm{U}}\right)$ & n.a. & n.a. & 19,29 & n.a. & n.a. & n.a. & n.a. & n.a. \\
\hline P-value (5 d.f.) & n.a. & n.a. & 0,00 & n.a. & n.a. & n.a. & n.a. & n.a. \\
\hline
\end{tabular}

Table 3 Test for differential response by education

Grouped over language, income (but controlled for in index)

Log lik High educated

Log lik Low educated

Log lik sum (unrestricted model, $\Lambda^{\mathrm{U}}$ )

Log likelihood restricted model $\left(\Lambda^{\mathrm{R}}\right)$

$-2 *\left(\Lambda^{\mathrm{R}}-\Lambda^{\mathrm{U}}\right)$

$\mathrm{P}$-value (8 degrees of freedom)

\begin{tabular}{rlrr}
\multicolumn{2}{c}{ Males } & \multicolumn{2}{c}{ Females } \\
Young & \multicolumn{1}{c}{ Old } & \multicolumn{1}{c}{ Young } & \multicolumn{1}{c}{ Old } \\
$-1459,44$ & $-2570,69$ & $-1668,38$ & $-3278,79$ \\
$-1563,34$ & $-1544,78$ & $-2035,27$ & $-1738,98$ \\
$-3022,78$ & $-4115,46$ & $-3703,64$ & $-5017,77$ \\
$-3025,74$ & $-4120,15$ & $-3709,71$ & $-5023,48$ \\
5,93 & 9,37 & 12,14 & 11,42 \\
0,65 & 0,31 & 0,15 & 0,18 \\
\hline
\end{tabular}


Table 4 Test for differential response by gender

Grouped over language, income, education

(controlled for in index)

\begin{tabular}{lrr} 
& \multicolumn{1}{c}{ Young } & \multicolumn{1}{c}{ Old } \\
\hline Log lik Males & $-4120,15$ & $-3025,74$ \\
Log lik Females & $-5023,48$ & $-3709,71$ \\
Log lik sum (unrestricted model, $\left.\Lambda^{\mathrm{U}}\right)$ & $-9143,63$ & $-6735,45$ \\
Log likelihood restricted model $\left(\Lambda^{\mathrm{R}}\right)$ & $-9159,17$ & $-6744,06$ \\
$-2 *\left(\Lambda^{\mathrm{R}}-\Lambda^{\mathrm{U}}\right)$ & 31,09 & 17,21 \\
P-value $(9$ degrees of freedom) & 0,00 & 0,05 \\
Log lik semi-restricted model $\left(\mathrm{Cut}-\mathrm{pnts}\right.$ free, $\left.\Lambda^{\mathrm{CP}}\right)$ & $-9154,60$ & $-6743,49$ \\
$\mathrm{X}^{2}$ test for cut-point shift $-2 *\left(\Lambda^{\mathrm{R}}-\Lambda^{\mathrm{CP}}\right)$ & 9,15 & 1,13 \\
P-value $(3$ d.f.) & $\mathbf{0 , 0 3}$ & 0,77 \\
$X^{2}$ test for index shift $-2 *\left(\Lambda^{\mathrm{CP}}-\Lambda^{\mathrm{U}}\right)$ & 21,94 & 16,08 \\
P-value $(7$ d.f.) & $\mathbf{0 , 0 0}$ & $\mathbf{0 , 0 2}$ \\
\hline
\end{tabular}

Table 5 Test for differential response by age

Grouped over language, income,education

(controlled for in index)

Log lik Old group

Males

Females

Log lik Young group

$-3025,74 \quad-3709,71$

Log lik sum (unrestricted model, $\Lambda^{\mathrm{U}}$ )

$-4120,15 \quad-5023,48$

Log likelihood restricted model $\left(\Lambda^{\mathrm{R}}\right)$

$-7145,89 \quad-8733,19$

$-2 *\left(\Lambda^{\mathrm{R}}-\Lambda^{\mathrm{U}}\right)$

$\mathrm{P}$-value (9 degrees of freedom)

$-7185,18 \quad-8748,75$

Log lik semi-restricted model (Cut-pnts free, $\left.\Lambda^{\mathrm{CP}}\right) \quad-7166,00 \quad-8740,54$

$\mathrm{X}^{2}$ test for cut-point shift $-2 *\left(\Lambda^{\mathrm{R}}-\Lambda^{\mathrm{CP}}\right) \quad 38,35 \quad 16,42$

P-value (3 d.f.)

$0,00 \quad 0,00$

$\mathrm{X}^{2}$ test for index shift $-2 *\left(\Lambda^{\mathrm{CP}}-\Lambda^{\mathrm{U}}\right) \quad 40,22 \quad 14,70$

P-value (7 d.f.) 


\section{References}

Crossley, T.F. and S. Kennedy, 2002, The relaiability of self-assessed health status, Journal of Health Economics, 21, 643-658

Cutler, D., Richardson, E., 1997. Measuring the health of the United States Population. Brookings Papers on Economic Activity, Microeconomics, 217-271.

Feeny, D., Furlong, W., Boyle, M., Torrance, G., 1995. Multi-attribute health status classification systems: Health Utilities Index. Pharmacoeconomics 7 (6), 490-502.

Feeny, D., Furlong, W., Torrance, G.W., Goldsmith, Zhu, Z C., DePauw, S.,., Denton, M., and Boyle, M., 2002. Multi-Attribute and Single-attribute Utility Functions for the Health Utilities Index Mark System, Medical Care 40(2):113-28

Gravelle H. (20010, 'Measuring Income Related Inequality in Health and health Care: the partial Concentration Index with Direct and Indirect Standardisation, Working Paper university of York.

Groot, W., 2000. Adaptation and scale of reference bias in self-assessments of quality of life. Journal of Health Economics 19(3), 403-420.

Hays, RD, LS Morales and SP Reise (2000). Item response theory and health outcomes measurement in the $21^{\text {st }}$ Century, Medical Care, 38 (9), Supplem II, II28- II42

Humphries, K and E van Doorslaer, 2000, Income-related inequalities in health in Canada, Social Science and Medicine, 50, 663-671

Jones, A.M., 2000. Health Econometrics, in A.J.Culyer and J.P.Newhouse (eds.) Handbook of Health Economics. Elsevier, Amsterdam.

Kerkhofs, M., Lindeboom, M., 1995, Subjective health measures and state dependent reporting errors. Health Economics 4, 221-235.

Murray, CJL, A Tandon, J Salomon, CD Mathers and R Sadana, 2001, Cross-population comparability of evidence for health policy, GPE Discussion Paper $\mathrm{Nr}$ 46, WHO/EIP, Geneva.

Sadana, R, CD Mathers, AD Lopez, CJL Murray and K Iburg, 2000, Comparative analysis of more than 50 household surveys on health status, GPE Discussion Paper No 15, EIP/GPE/EBD, World Health organisation, Geneva.

Sen, A, 2002, Health: perception versus observation, British Medical Journal, 324, 660-1

Shmueli, A, 2002, Reporting heterogeneity in the measurement of health and health-related quality of life, Pharmacoeconomics, 20 (6), 405-412

Shmueli, A, 2003, Socio-economic and demographic variation in health and in its measures: the issue of reporting heterogeneity, Social Science \& Medicine, 57, 125-134

Tambay J-L., Catlin, G., 1995. Sample Design of the National Population Health Survey. Health Reports 7(1), 29-38.

Tandon, A, CJL Murray, JA Salomon and G King, 2002, Statistical models for enhancing cross-population comparability, Global Programme for Evidence on Health Policy Discussion Paper Nr. 42, WHO, Geneva. 
Torrance, G.W., Furlong, W., Feeny, D., Boyle, M., 1995. Multi-attribute preference functions: Health Utilities Index. Pharmacoeconomics 7(6), 503-520.

Torrance, G.W., Feeny, D., Furlong W.J., Barr, R.D., Zhang, Y., Wang, Q., 1996. Multiattribute Utility Function for a Comprehensive Health Status Classification System. Medical Care 34(7), 702-722.

Van Doorslaer, E., Wagstaff, A., Bleichrodt, H., et al, 1997. Income-related inequalities in health: some international comparisons. Journal of Health Economics 16, 93-112.

Van Doorslaer, E and AM Jones, 2003, Inequalities in self-reported health: validation of a new approach to measurement, Journal of Health Economics, 22, 61-87

Van Doorslaer, E. \& U. Gerdtham (2003), 'Does inequality in self-assessed health predict inequality in survival by income? Evidence from Swedish data', forthcoming in Social Science \& Medicine.

Wagstaff, A., van Doorslaer, E., 1994. Measuring inequalities in health in the presence of multiple-category morbidity indicators. Health Economics 3, 281-291. 


\section{Appendix}

Table A1: Descriptive statistics

\begin{tabular}{lcc}
\hline Variables & Mean & Std. Dev \\
Health Utility Index & 0.893 & 0.130 \\
SAH excellent & 0.237 & 0.425 \\
SAH very good & 0.384 & 0.486 \\
SAH good & 0.265 & 0.441 \\
SAH fair & 0.091 & 0.287 \\
SAH poor & 0.024 & 0.152 \\
Speaks French only & 0.088 & 0.283 \\
Speaks both English and French & 0.147 & 0.354 \\
Speaks other language & 0.147 & 0.354 \\
Female & 0.550 & 0.498 \\
Low education & 0.532 & 0.499 \\
Young $(<35 y)$ & 0.486 & 0.500 \\
Low income $(<$ Can $\$ 24748$ per eq adult $)$ & 0.497 & 0.500 \\
\hline
\end{tabular}

Table A2: Mean HUI values by level of SAH and background characteristics

\begin{tabular}{llccccc}
\hline \multirow{2}{*}{ By: } & & \multicolumn{5}{c}{ Self-assessed health } \\
Sex & male & Poor or fair & Good & Very good & Excellent & Total \\
& female & 0.736 & 0.882 & 0.928 & 0.946 & 0.900 \\
Age & old & 0.715 & 0.871 & 0.920 & 0.944 & 0.888 \\
& young & 0.754 & 0.864 & 0.914 & 0.934 & 0.869 \\
Education n & high & 0.727 & 0.893 & 0.931 & 0.953 & 0.918 \\
& low & 0.722 & 0.872 & 0.928 & 0.947 & 0.909 \\
Income & high & 0.743 & 0.882 & 0.926 & 0.942 & 0.879 \\
& low & 0.716 & 0.872 & 0.921 & 0.945 & 0.909 \\
Language & English & 0.720 & 0.873 & 0.923 & 0.946 & 0.877 \\
& French & 0.735 & 0.882 & 0.930 & 0.941 & 0.892 \\
& both & 0.737 & 0.881 & 0.925 & 0.944 & 0.903 \\
& other & 0.722 & 0.880 & 0.923 & 0.945 & 0.891 \\
Total & & 0.724 & 0.876 & 0.923 & 0.945 & 0.893 \\
$\mathbf{N}$ & & 1567 & 3627 & 5265 & 3240 & 13699 \\
\hline
\end{tabular}

